\title{
Prendre le temps
}

\section{Take the time}

\section{Roegiers}

(C) Lavoisier SAS 2019

L'idée d'ouvrir le dossier du " Temps » est issue d'une complainte bien connue : "nous n'avons pas le temps; nous n'avons plus le temps ». Ce constat pourrait stupéfier un extraterrestre contemplant l'évolution des sapiens : arrivés au stade de la sophistication des outils destinés à alléger leur quotidien de chasseurs-cueilleurs et à maîtriser leur santé générale et reproductive, les sapiens ont pourtant le blues. Nous aurions perdu en chemin la faculté de jouir du temps et de la sécurité gagnés par la révolution technologique. La qualité de vie ne suit pas, c'est vrai partout et en particulier dans les hôpitaux, surtout dans des services à flux tendu et soumis aux aléas tels que la maternité ou le service néonatal. La quantité de temps fait encore et toujours défaut mais aussi sa qualité. Une étude sur le bien-être dans les néonatalogies intensives révèle l'importance de postures pour échapper au burn-out ou au stress post-traumatique des professionnels. La qualité du temps y est centrale : temps de découverte stimulante, temps de soins aux bébés en partenariat avec leurs parents, temps d'échanges avec des proches pour partager les émotions fortes, temps «pour soi », moments de repos et des choses qu'on aime [1]. Si telle est la recette de la résilience pour les professionnels vivant l'immersion en émotions concentrées, pourquoi ne serait-elle pas identique du côté des patients, des parents, des bébés ? Qu'en est-il de l'expérience du temps pour le couple lors du diagnostic prénatal, pour la femme en salle de naissance puis en furtif passage à la maternité, pour le bébé prématuré à la recherche du temps perdu?

Le constat de Francis Puech est implacable, nous souffrons de ne plus avoir le temps.

Or, la durée d'une grossesse est incompressible. Les limites en sont immuables, mais le séjour en maternité s'amenuise avec un cahier des charges sous tension.

\section{Roegiers ( $\bowtie)$}

Cliniques universitaires Saint-Luc,

10, avenue Hippocrate, B-1200 Bruxelles, Belgique

e-mail : luc.roegiers@uclouvain.be

GiP, Docteure-Marie-Laure Gustin,

27B, avenue Brugmann, B-1060 Bruxelles, Belgique
Les plans périnatalité des années 1990 étaient ceux de l'espace : hiérarchisation des centres et organisation des transferts ; les plans des années 2000 furent ceux de la temporalité : entretien prénatal précoce (EPP), anticipation... Ces initiatives ont soulevé beaucoup d'espoirs, parfois suivis de déceptions. L'EPP reste limité par manque d'attention aux autres professionnels, par un exercice solitaire trop crispé sur l'instant présent et pas assez sur la transmission, par la perte du fil médical dans certaines situations complexes. Le PRADO à l'autre extrême de la chaîne cette fois visait l'anticipation du retour à domicile... on en a retenu la réduction du séjour et trop peu la temporalité nécessaire à la triade mère-père-nouveau-né... Francis Puech conclut par une réanimation des objectifs déjà énoncé « humanité, proximité, sécurité, qualité » en proposant plusieurs pistes malgré le facteur « temps » devenu un handicap plus qu'une aide.

L'enjeu en vaut la peine, comme nous le rappelle MariePaule Lacourt-Mrozek dans son article témoignant d'un patient tissage interdisciplinaire dans son bassin de soins périnataux. Le temps investi est toujours valorisé en prévention.

Quand on parle du temps, c'est souvent en termes de frustration : «pas assez de temps » ou « un temps insupportable avant d'être entendue ». Deux extrêmes de souffrance d'un temps non ajusté.

À un bout, l'expérience du stress est étroitement liée à la contraction du temps. C'est étymologique, le «stress » vient du latin stringere, avec pour exemple du Larousse : il tempo stringe... « le temps presse ». «Être pressé » peut signifier à la fois avoir trop de choses à faire en trop peu de temps ; être exploité, pressé comme un citron ; ou encore être dos au mur. Bref des circonstances désagréables. À l'autre extrême de l'expérience du temps, il y a le malaise inverse : l'angoisse existentielle de la solitude, du vide,... l'antichambre du néant. À remplir donc d'activités. L'humain n'est pas en manque à cet égard, et la multiplication des outils, media, jeux, techniques, transports... aboutit aujourd'hui à une temporalité lotie de « choses à faire ». Particulièrement, dans le domaine médical, on ne manque pas de gadgets - monitos, images, outils, protocoles... - qui parfois sauvent des vies 
et parfois sont les bouche-trous de nos impuissances anxieuses, avec un impact variable sur l'usager. Les opposés se touchent, l'angoisse pousse à remplir le temps jusqu'au stress à son tour générateur d'angoisse.

Dommage ; et si on abordait le temps par son versant positif? Le temps nous enveloppe, nous porte et nous oxygène. Voilà les premiers mots d'une journée d'études du groupe belge interdisciplinaire/interuniversitaire de périnatalité en février 2018. Plusieurs textes de ce dossier en sont issus.

Pour vivre comme un poisson dans l'eau disait l'argumentaire, nous devons relever plusieurs défis « temporels » en période périnatale :

- réconcilier deux sortes de temps, face objective et face subjective : Jean-Michel Longneaux les distingue ainsi :

- le premier temps est impersonnel, prévisible ; c'est celui du chrono, de la maîtrise et du contrôle versus l'autre temps créateur de nouveauté, personnalisé, intervenant « à l'improviste »;

- la succession des phases biologiques - en causeseffets, temporalité stricte des processus de soins, organisation des relais, traçabilité... versus un bonheur ou un malheur concernant quelqu'un en particulier ; on touche là le hasard et l'imprévisibilité du vivant, avec toujours la dimension créative. On « tombe » malade, amoureux,..., enceinte, dit le philosophe Jean-Michel Longneaux.

Combien de clashs résultent-ils du décalage entre ces deux types de temps? Les professionnels organisent leur temps, les usagers font l'expérience d'un moment de leur vie, un événement majeur lorsqu'il s'agit de leur enfant. Comme ils le peuvent, les usagers essaient par leur projet de naissance ou par d'autres accès de s'approprier ce temps, sans préjudice des protocoles ;

- offrir aux parents et au bébé l'expérience de la continuité... Comment articuler les temps et les intervenants des grossesses à risque ou du diagnostic prénatal à ceux de la maternité et de la néonatologie ? Comment anticiper les besoins de façon à atténuer le choc de l'adaptation après l'accouchement en cas de vulnérabilité somatique, ou de vulnérabilité psychique ? Comment passer d'une rencontre à l'autre dans des transitions et des délais rassurants ? C'est l'objectif des professionnels, des parents et... des bébés. En gros plan, nous pouvons voir sur écran l'adaptation laborieuse d'un nouveau-né grand prématuré face à diverses interventions de soignants, à des modifications de posture... Son défi est de reprendre son souffle, de retrouver son expérience de stabilité sécurisante, de récupérer sa fluidité. L'harmonie du temps produit la compétence. Marie-Françoise Livoir Petersen en fait son propos dans son article sur le temps des soins de développement. Il est transposable à bien d'autres situations de crise ;
- il faut en même temps faire face aux impératifs de « rapidité-efficacité »... faire face aux priorités médicales... forcer le temps quand il n'y a plus une minute à perdre : deux études contradictoires apparaissaient au même moment dans la littérature périnatale. L'une met en exergue la nécessité de laisser au travail le temps de se déployer pour respecter la physiologie [2]. L'autre relie la durée d'effectuation d'une césarienne urgente et son issue [3]. Les usagers comprennent certaines intrusions violentes en situation critique. On peut reprendre et justifier la succession des gestes. Ils laissent parfois une trace psychotraumatique, mais cela se travaille alors que certains dégâts somatiques s'avèrent irréversibles. Ce que ne supportent ni les usagers ni les équipes médicales et soignantes, c'est l'impératif économique de l'accélération du temps. Jusqu'où accepter le diktat du Time is money? Et ne perdons-nous pas parfois beaucoup de temps à essayer d'en gagner un peu ? Les professionnels de la santé peuvent être généreux en temps offert, à condition qu'ils s'en sentent gestionnaires et reconnus ; sinon c'est l'énervement garanti, et parfois une fameuse pagaille chronophage. Le documentaire burning-out tourné à Saint-Louis en est la meilleure illustration. Lisez à ce sujet dans notre dossier le texte d'un économiste de la santé pour reprendre de l'oxygène. Christian Léonard travaille dans un centre belge d'expertise médicale entourant les politiques et témoigne qu'il s'y trouve des îlots de pensées sur le sens et la valeur du care, prendre soin au-delà de l'utilitarisme flamboyant qui nous tue au petit feu de la dépression, « le burn-out aux effets dramatiques quand il frappe les gens qui ont choisi de prendre soin des autres $» .$. « Accepter la logique incitative, dit Christian Léonard, c'est sans s'en rendre compte, concéder que l'humain est un être utilitariste pour qui le temps donné est du temps perdu, pour qui l'empathie et la bienveillance sont réservées aux "faibles" ";

- y aurait-il encore de la place pour le rythme des patients ? Les paroles de l'accouchement ne risquent-elles pas d'aller plus vite que sa musique ? Comment permettre à chacun l'assimilation des événements... C'est le dernier groupe de questions, articulées aux précédentes. Pouvons-nous en tant que soignants, sans préjudice de la sécurité médicale, faire place au temps de la maman, à celui du bébé ? Anne Evrard nous propose une histoire d'usagère soumise en diagnostic prénatal à la contraction de sa grossesse. Cette mère cherchait à réinvestir cette trop courte durée, une invitation à lui laisser le temps. Elle était télescopée entre FISH et IMG. Attention, rappelle Anne Evrard, les parents adhèrent par défaut au rythme proposé par les protocoles, habitudes et soucis organisationnels... ce fameux temps standardisé, pas forcément ajusté. Lorsqu'on peut valider qu'il n'y a pas d'urgence, on peut être attentif au rythme des usagers, leur ouvrir des 
possibilités de choisir... Du coup, la confiance mutuelle rend plus facile le déroulement là où le professionnel peut avoir une peur d'éparpillement.

À propos du temps vécu par les uns et par les autres, cet éditorial serait incomplet s'il ne rappelait l'importance de la temporalité dans nos reprises de situations cliniques lors des revues de morbidité-mortalité ou lors d'analyses interdisciplinaires selon la méthode prospective proposée par Françoise Molénat et son équipe. Pour tirer de façon formative les enseignements de nos réussites et de nos échecs, il faut repasser les étapes temporelles traversées par chaque acteur de la situation : ses interrogations à tel moment, puis ses collaborations, ses concertations, ses transmissions, ses outils, ses relais et supports. Les détails et les questions posées en progressant pas à pas dans la reprise sont plus éclairants et instructifs qu'une synthèse reconstruite a posteriori. Et surtout, les « arrêts sur image » vécus par les usagers aident à comprendre comment ils ont pu habiter tels moments de telle situation, comment ceux-ci ont pris place dans leur histoire.

L'échographiste Michel Van Rysselberge a reconstitué dans son article, du côté professionnel et du côté des usagers, le déroulement d'une histoire singulière, de celles qui marquent une vie. Il nous parle du rythme assimilable, du souci d'anticiper, du temps présent parfois insupportable, du sens à trouver, de l'ajustement permanent entre le temps du professionnel et le temps de la mère. Michel Van Rysselberge nous invite au cœur du voyage intérieur d'une de ses patientes, de son expérience du temps dans une terre complètement inconnue, expérience renforcée par sa réalité d'immigrée. Son pays est perdu dans les temps anciens auxquels ses parents tentent de la reconnecter par des remèdes traditionnels, un éprouvé « connu » dans leur mémoire culturelle... lorsque les protocoles invitent à traverser bien trop vite l'épreuve. Mais ici, le gynéco-obstétricien a anticipé. Il laisse à cette patiente le temps de souffler, de se préparer dans la mesure du possible. La suite des événements se déroule de façon accélérée, de plus en plus instable. Mais la chaîne des intervenants est là. Les fils du temps différents des acteurs - le fil de la suite d'actes techniques définis, le fil hésitant de l'errance diagnostique, le fil tendu du temps impatient et impuissant du mari, le fil de sollicitude parfois décalée « à contretemps » de l'entourage familial, le fil des bébés dont l'essence est d'être en devenir ou en non-devenir..., ces fils enchevêtrés doivent être identifiés et démêlés pour être retissés en une cocréation contenante dans laquelle l'enfant sera sécurisé quelle qu'ait été l'intensité dramatique du voyage qui le mène à la vie.

\section{Références}

1. Rippstein-Leuenberger K, Mauthner O, Bryan Sexton J, Schwendimann R (2017) A qualitative analysis of the Three Good Things intervention in healthcare workers. BMJ Open 7:e015826. doi:10.1136/bmjopen-2017-015826

2. Henderson J, Redshaw M (2017) Sociodemographic differences in women's experience of early labour care: a mixed methods study. BMJ Open 7:e016351. doi:10.1136/bmjopen-2017-016351

3. Heller G, Bauer E, Schill S, et al (2017) Decision-to-delivery time and perinatal complications in emergency cesarean section. Dtsch Arztebl Int 114:589-96. doi: 10.3238/arztebl.2017.0589 